\title{
Estimating the Fatality Burden of SARS-CoV2
}

\author{
BARRY SMYTH, Insight Centre for Data Analytics, School of Computer Science, University College Dublin,
} Ireland

As the world struggles with the SARS-CoV2 pandemic, public health officials and governments continue to refine the key metrics that are used to capture and compare the state of the pandemic and the effects of responses within and between countries and regions. This work presents a novel fatality metric, the COVID-19 burden, which normalises SARS-CoV2 fatalities with respect to historical mortality rates over the same period of time. We argue that this measure provides an improved basis for comparing fatality rates between countries, and we present an analysis of this measure across 174 countries, using data up to November 15, 2020, to better understand the impact of the virus in different countries and regions.

CCS Concepts: • Applied computing $\rightarrow$ Health informatics;

Additional Key Words and Phrases: Data science, COVID-19

ACM Reference format:

Barry Smyth. 2020. Estimating the Fatality Burden of SARS-CoV2. Digit. Gov.: Res. Pract. 2, 2, Article 21 (December 2020 ), 8 pages.

https://doi.org/10.1145/3436997

\section{INTRODUCTION}

As the global spread of SARS-CoV2 continues [10,19], many countries have entered a new phase in their struggle with the virus $[1,3,5,8,16,17,20]$. Spring lockdowns reduced the prevalence of the virus in many locations [2, 15], but as economies re-open incidence rates are rising again, and the risk of infection, long-term illness, and even death remains an ever-present one, especially if the virus manages to break through to more vulnerable groups, as it did in March and April [11].

It is important to equip public health officials with the tools and techniques they need to monitor and manage outbreaks $[4,7,15,18]$. This includes the development of meaningful comparative metrics to identify countries and regions that have performed well, or less well, so lessons can be learned from the approaches they have tried. In this work, we present a analysis of COVID-19 fatalities from 174 countries around the world using a pair of novel fatality metrics, the COVID-19 burden and the COVID-19 rank, which normalise COVID-19 deaths with respect to historical all-cause mortality statistics. We argue that they provide a fairer way to compare the impact of the virus between different countries, given that historical mortality rates can vary considerably.

Supported by Science Foundation Ireland through the Insight Centre for Data Analytics under grant 12/RC/2289P2.

Author's address: B. Smyth, Insight Centre for Data Analytics, School of Computer Science, University College Dublin, Belfield, Dublin 4 Ireland; email: barry.smyth@ucd.ie.

Permission to make digital or hard copies of all or part of this work for personal or classroom use is granted without fee provided that copies are not made or distributed for profit or commercial advantage and that copies bear this notice and the full citation on the first page. Copyrights for components of this work owned by others than ACM must be honored. Abstracting with credit is permitted. To copy otherwise, or republish, to post on servers or to redistribute to lists, requires prior specific permission and/or a fee. Request permissions from permissions@acm.org.

(c) 2020 Association for Computing Machinery.

2639-0175/2020/12-ART21 $\$ 15.00$

https://doi.org/10.1145/3436997

Digital Government: Research and Practice, Vol. 2, No. 2, Article 21. Publication date: December 2020. 


\section{ALL-CAUSE MORTALITY NORMALISED SARS-COV2 FATALITY METRICS}

The proposed COVID-19 burden and rank metrics adjust fatalities with respect to national, historical mortality statistics, and therefore normalise COVID-19 deaths using a host of latent mortality factors. However, caution is required when interpreting these metrics due to inconsistencies in how countries account for, and report on, COVID-19 deaths.

\subsection{The COVID-19 Burden}

The COVID-19 burden is defined by Equation (1): $f$ is the total number of COVID-19 fatalities in a country/region $(C)$ since the first reported COVID-19 fatality; $e$ is the expected number of all-cause annual deaths for a country/region; and $d$ is the number of days since the first reported COVID-19 fatality.

$$
\operatorname{burden}_{C}(f, e, d)=\frac{f}{d} \times \frac{365}{e}
$$

For example, on November 15, 2020, it was 248 days since Ireland's first reported COVID-19 death and there were 1,978 COVID-19 deaths in that period. According to the Irish Central Statistics Office, ${ }^{1}$ in 2017 there were 30,418 deaths in Ireland, or approximately 20,667 in a 248 -day period. Therefore, Ireland's current COVID-19 burden is approximately 0.095 ; that is, COVID-19 deaths represent just over $9 \%$ of the expected (historical) deaths during the same period of time, or just over $6 \%$ of the expected deaths for a full year.

We argue that this approach offers significant advantages over conventional fatality measures, such as the number of deaths per capita, because it normalises with respect to a variety of latent mortality factors, such as population demographics, the incidence of co-morbidities, healthcare quality and accessibility, food security, and so on. Thus, two similarly sized countries, which might ordinarily be judged to have similar fatality rates, if their deaths per capita are similar, will be recognised to be different, if their historical mortality rates diverge, and their COVID-19 burdens will reflect this difference.

\subsection{The COVID-19 Rank}

The related COVID-19 rank metric estimates the rank of COVID-19, as a cause of death, by comparing COVID-19 deaths with the historical causes of death in a country. For example, in Ireland the top-five causes of death are cardiovascular disease (9,600 deaths per year), cancers (9,500 deaths), dementia (2,700 deaths), respiratory diseases (2,225 deaths), and lower respiratory infections (1,371 deaths). This means that Ireland's current 1,978 COVID-19 deaths, ranks COVID-19 as Ireland's 5th highest cause of death; thus, its COVID-19 rank is 5, as of November 15, 2020.

We can also calculate a relative rank version of this metric, such that a relative rank of 0 indicates the topranking cause of death, while a relative rank of 1 corresponds to the lowest-ranking cause of death. This is useful when comparing countries that have different numbers of causes of death. The relative rank of COVID-19 in Ireland is 0.17 , as it is 5 th out of 29 common causes of death.

\subsection{Caveats}

There are two important caveats to this approach. First, it assumes a uniform distribution of deaths over time for a country, which is likely to be incorrect over short periods of time (weeks), but given that the pandemic has been in progress for most of the year, this is not likely to be a major source of error.

Reporting inconsistencies between countries are another matter. Some countries only include deaths that arise from a confirmed COVID-19 test, others have limited their focus to hospital settings, while yet others have been

\footnotetext{
${ }^{1}$ https://www.cso.ie/en/releasesandpublications/ep/p-vsar/vitalstatisticsannualreport2017/deaths2017/.
} 


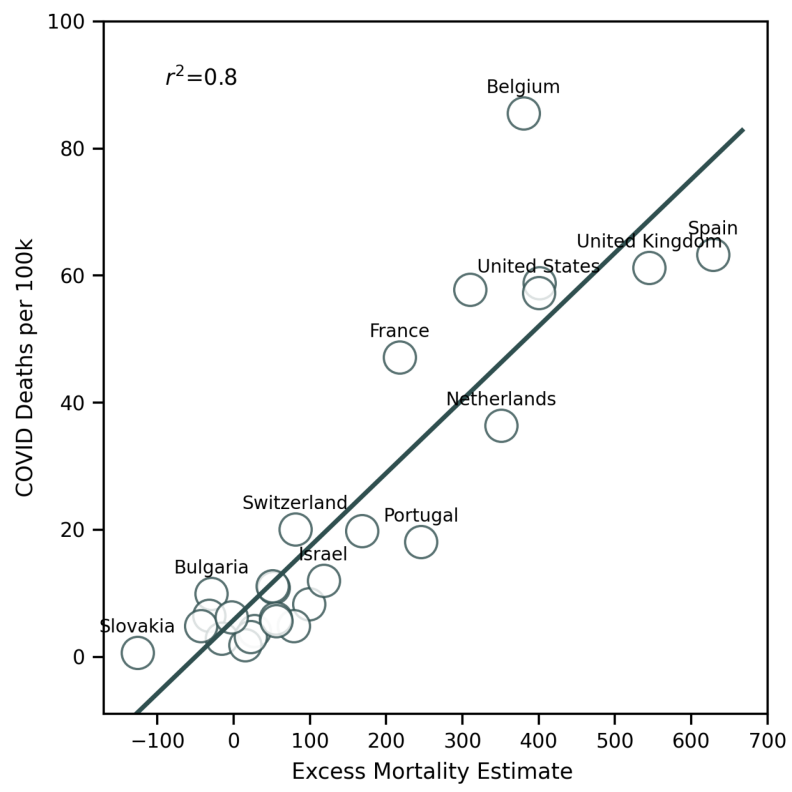

Fig. 1. Comparing official COVID-19 fatalities and excess mortality estimates using a dataset of 27 countries.

far more inclusive, counting suspected and confirmed deaths across all settings. To evaluate the scale of such inconsistencies, we can compare official COVID-19 fatality statistics to the so-called excess deaths for a country. The number of excess deaths for a country provides an estimate of the additional deaths this year, regardless of cause, by comparing total numbers of deaths in 2020 to historical averages. While this is viewed as a more accurate COVID-19 fatality estimate, excess death data is not widely available at this time.

Figure 1 shows the relationship between population-normalised COVID-19 deaths (COVID-19 deaths per 100,000 of population) and excess deaths for a dataset [6] of 27 countries with data availability up to the end of September 2020. There is a strong relationship between COVID-19 fatalities and excess deaths $\left(r^{2}=0.8\right)$, but not a perfect one. Note, excess deaths are measured as a sum of the daily percentage differences between current and historical deaths; this is the excess mortality estimate used for the $\mathrm{x}$-axis in Figure 1. For example, Belgium had a total daily excess of $400 \%$ for the COVID-19 period up to the end of September, based on an average daily excess of approximately $2.5 \%$; for the avoidance of doubt, this does not mean that Belgium has had four times $(400 \%)$ as many deaths as usual, it means there has been an average daily excess of just $2.5 \%$, in that period, above and beyond historical figures.

Most countries fall close to the linear regression line used to estimate the relationship between these two fatality measures, but Belgium is an obvious outlier, at least up to the end of September 2020. Its official COVID-19 fatalities are much higher than other countries with similar excess deaths in this time period, which suggests its COVID-19 fatality estimate might need to be reduced (by $\approx 40 \%$ ) to bring it into line with the reporting standards of others, and such an adjustment will obviously have a corresponding effect on Belgium's COVID-19 burden. In a similar way, the UK's estimate may need to be increased by approximately $10 \%$.

Since the degree of this variation between countries is modest ( $\leq 20 \%$ on average), it means that reporting inconsistencies between countries are likely to be modest, too. However, caution should be exercised when comparing countries to one another, especially if their COVID-19 fatalities differ by $<20 \%$, because such differences may be an artefact of reporting rather than a genuine difference in the number of COVID-19 related deaths.

Digital Government: Research and Practice, Vol. 2, No. 2, Article 21. Publication date: December 2020. 


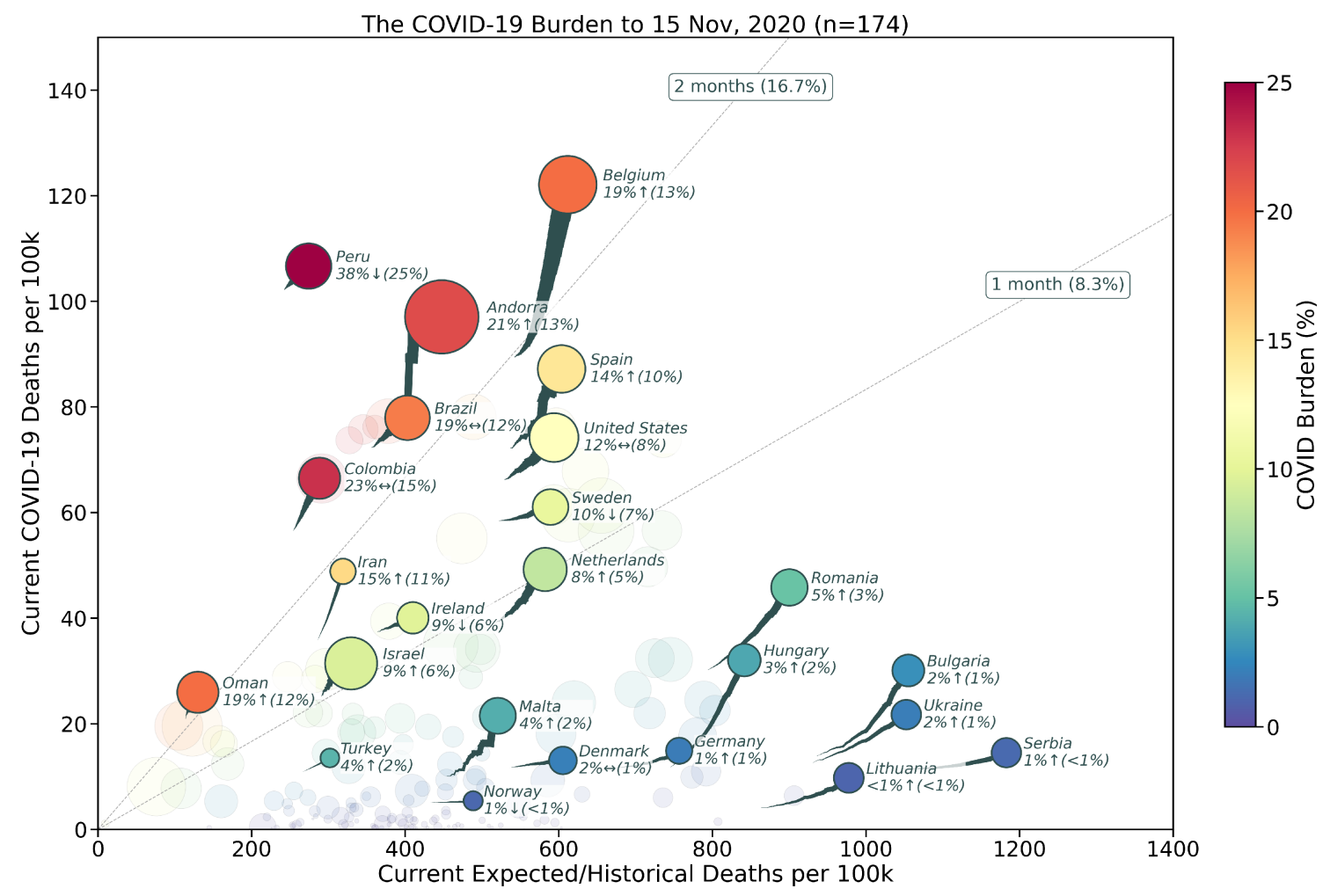

Fig. 2. A comparison of countries based on their current official COVID-19 fatalities and expected deaths using historical all-cause mortality data per 100,000 of population. The size of the marker is proportional to the total number of confirmed cases per 100,000 of population and each marker is coloured to reflect its current COVID-19 burden. Each marker's tail indicates its trajectory over the past 30 days.

\section{AN ANALYSIS OF COVID-19 BURDEN}

In what follows, we present a summary analysis of the COVID-19 burden and ranks for a publicly available dataset [14] of daily COVID-19 cases and deaths, and by using a second public dataset [13] of historical all-cause mortality statistics; the former dataset includes data up to and including November 15, 2020.

\subsection{Dataset \& Methodology}

These datasets include data for 174 countries. For each country and date we calculate the COVID-19 burden and rank. We also calculate the current COVID-19 burden trend, based on the relative change in COVID-19 burden compared with 30 days previously. In what follows, we compare the current burdens and ranks by country and continent.

\subsection{The COVID-19 Burden by Country}

Figure 2 shows a plot of these 174 countries, in terms of their current total COVID-19 deaths and the expected number of deaths, based on historical mortality statistics over the same period (that is, up to November 15 , 2020). Each marker represents a single country. Its size is proportional to the total number of confirmed cases per 100,000 of population and the colour of the marker reflects its current COVID-19 burden, according to the scale shown. Each marker is also associated with a "tail," which reflects the trajectory of the country's COVID-19 
deaths over the past 30 days. Thus, a horizontal tail indicates that a country has accumulated no new COVID-19 deaths over the past 30 days, whereas an upwardly sloping tail indicates increasing numbers of COVID-19 deaths over the past 30 days.

The highlighted countries are also annotated with their current COVID-19 burden, expressed as a percentage, and an arrow indicating whether the burden trend has been increasing or decreasing. The second percentage shown is a version of burden that is calculated from the current COVID-19 deaths but with respect to a full year of expected deaths. For example, Ireland has a current (November 15, 2020) COVID-19 burden of 9\%, which is falling, and currently COVID-19 deaths represent $6 \%$ of a full year of expected deaths.

The guidelines shown on the graph help to highlight different clusters of countries based on their COVID-19 burden trajectories. One guideline represents a burden of just over $8.3 \%$, which corresponds to a single month of annual deaths, and just under $79 \%$ of countries have a burden that is currently less than this. At the other extreme, there is a subset of 12 countries (just under 7\%), many from Central and South America, with a burden that is in excess of $16.6 \%$ (or two full months' worth of expected deaths) highlighting the seriousness of the outbreaks in those regions. ${ }^{2}$ The middle-ground (between one and two months' worth of deaths) is occupied by 25 countries from around the world. ${ }^{3}$

As many countries now enter a new COVID-19 wave during the winter months, we can see how some are struggling with an increasing COVID burden. For example, in Europe, Belgium, and Spain, already hard-hit during the first wave, are once again under pressure, as indicated by their steeply sloping tails, signalling a sharp increase in the number of COVID-19 deaths over the past 30 days. Likewise, the tails of countries such as Bulgaria, the Netherlands, and Sweden are also beginning to swing upwards. In contrast, many other European countries (e.g., Norway, Denmark, Germany, Ireland) appear to be coping more effectively with their second wave, as indicated by tails that are almost horizontal or only gently sloping upwards.

\subsection{The COVID-19 Rank by Continent}

Figure 3 summarises the COVID-19 rank and burden at the continent level, along with a variety of related information, as follows:

(1) The bar graph shows the mean/standard deviation of relative COVID-19 ranks, averaged over all countries in a given continent, and with each bar annotated by the average actual rank for each continent.

(2) The colour of each bar is based on the mean COVID-19 burden, and this value and the burden trend are also indicated by the text annotation.

(3) Separately, the y-axis position of the line graph encodes the average duration of outbreaks (days) by continent, and the size of each continent marker is proportional to the mean number of cases per 100,000 of population for the countries of that continent.

(4) The number above each marker indicates the number of countries in the corresponding continent in our dataset, and the numbers inside each marker indicate the actual number of cases per 100,000 and the mean case fatality rate for that continent.

The high impact of the virus in South America is clearly evident. There, outbreaks have been proceeding for about 238 days, and the 12 countries included currently have an average case load in excess of 1,500 cases per 100K of population, with a corresponding case fatality rate of 0.03 . Deaths in South America represent approximately $14 \%$ of historical deaths during the period, making COVID-19 the 7th highest cause of death, to date, out of 30 common causes of death.

\footnotetext{
${ }^{2}$ As of November 15, 2020, the full list of countries in this cluster is: Andorra, Belgium, Bolivia, Brazil, Chile, Colombia, Ecuador, Kuwait, Mexico, Oman, Panama, Peru.

${ }^{3}$ This cluster includes: Argentina, the Czech Republic, France, Iran, Iraq, Ireland, Israel, Italy, Jordan, Netherlands, Saudi Arabia, Spain, Sweden, United Kingdom, and the United States, among some others.
}

Digital Government: Research and Practice, Vol. 2, No. 2, Article 21. Publication date: December 2020. 


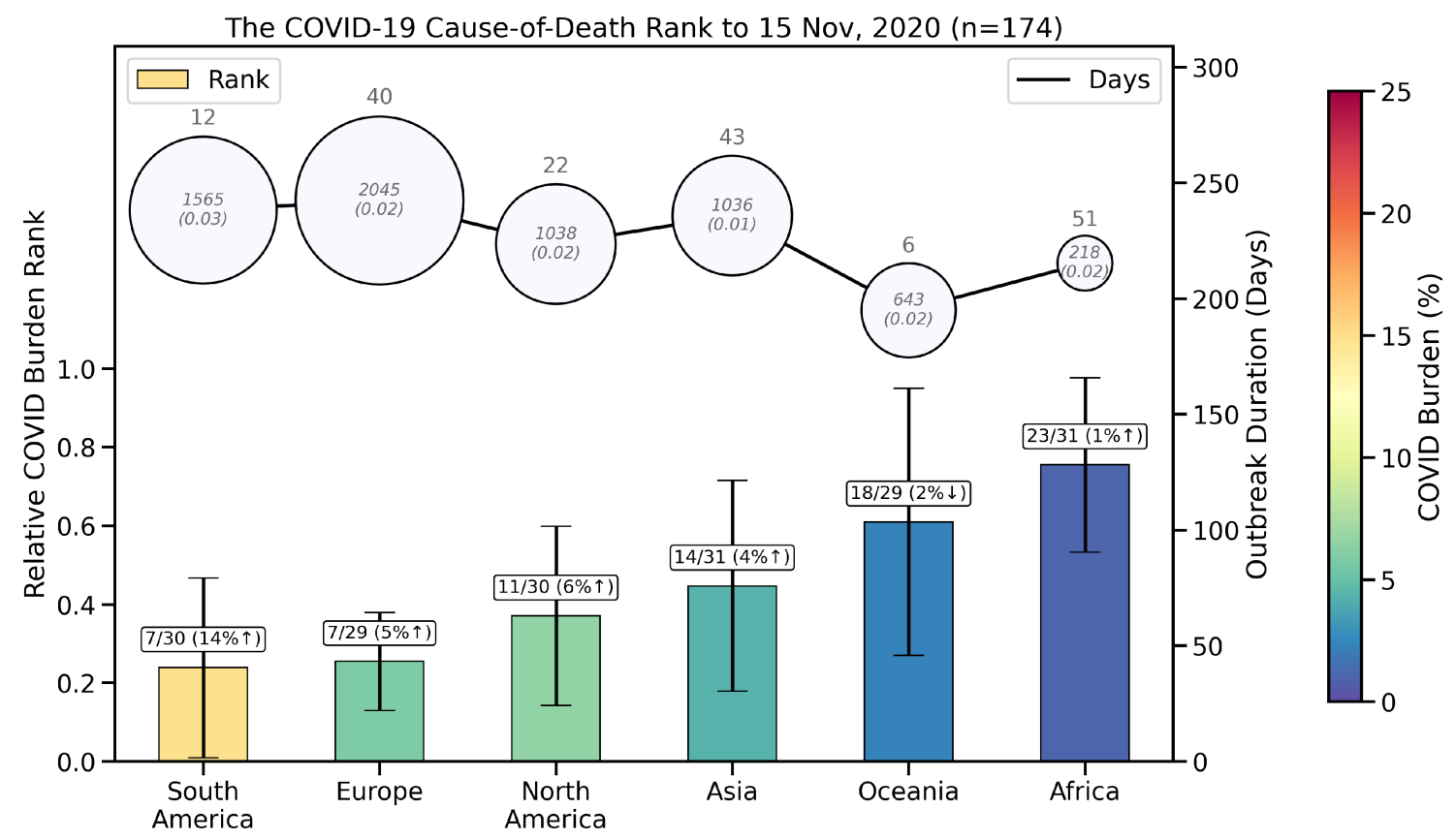

Fig. 3. The average relative rank of COVID-19 per continent (bar graph) and average scale and duration of outbreaks (line graph).

The scale of Europe's outbreak is greater than that of North America, 2,045 cases vs. 1,083 cases per 100K, on average per country, respectively; although, we must remember that countries such as the United States have far larger outbreaks than most in North America, hence the higher standard deviation indicator bars for North America compared with Europe. Both continents have similar case fatality rates (0.02), but North America has a higher COVID-19 burden (6\% compared with 5\% for Europe). This makes COVID-19 the 7th highest cause of death in Europe, compared with the 11th highest in North America. At the time of writing, Oceania is the only continent where there has been an average decrease in the COVID-19 burden over the last 30 days.

The numbers from Africa tell of a very different COVID-19 experience, compared with much of the rest of the world. Outbreaks there have been proceeding for just over 220 days, with an average of 218 confirmed cases per $100 \mathrm{~K}$ people, and a case fatality rate of 0.02. COVID-19 deaths in Africa account for just $1 \%$ of the historical deaths over the same period, making COVID-19 the 23rd highest cause of death in Africa. Not surprisingly, the status of SARS-CoV2 in Africa has been the subject of considerable discussion [9]. The consensus appears to be that the continent's low cases and deaths cannot be explained away as a reporting issue, although there is likely to be a wider margin of reporting error than elsewhere. One hypothesis [12] is that Africa's current success is due in part to population age-we know that COVID-19 fatalities are far more common in older age groups and the median age in Africa is just under 20 years old compared with 39 for the US-but Africa's success may also be linked to what its people have learned from the many previous pandemics that they have had to live through.

For completeness, Figure 4 shows the COVID-19 burden and rank for the countries used in this study, contrasting current hot-spots in South America to much milder and more contained outbreaks in Africa, Asia, and Oceania.

Digital Government: Research and Practice, Vol. 2, No. 2, Article 21. Publication date: December 2020. 
COVID-19 Burden and Cause-of-Death Rank to 15 Nov, $2020(n=174)$

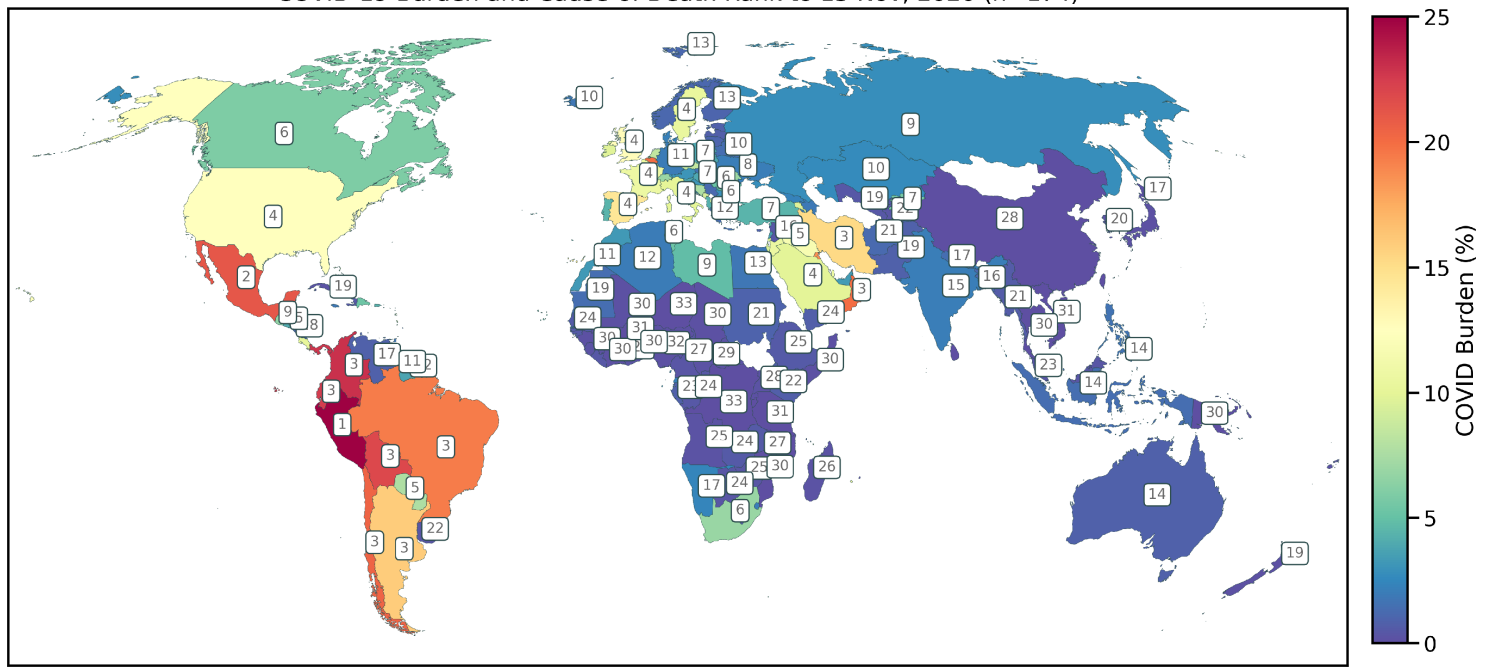

Fig. 4. Countries are colour-coded based on their COVID-19 burden and with selected (larger) countries annotated by their corresponding COVID-19 rank.

\section{CONCLUSIONS}

At the time of writing, early signs of the availability of safe and effective vaccines are beginning to be reported, but it will require months to scale up production and roll out, meaning that this pandemic will likely be with us through most of 2021, if not beyond. Therefore, it will be increasingly important for countries to develop coping strategies that are more tailored and more focused than past lockdowns, so a better balance may be achieved between public health and economic stability. Accurate and real-time data will be an important part of this effort, and the right metrics will be necessary to extract value from these data to determine the right response at the right time.

The COVID-19 burden and rank metrics are examples of a new type of metric that are better adapted to understanding this pandemic. By normalising COVID-19 fatalities with respect to historical mortality rates, these metrics provide a more representative account of COVID-19 fatalities across the world. In this work, we have defined these metrics and used them to compare fatality levels in 174 countries around the world to show how different regions and countries have had very different COVID-19 experiences. The approach taken may also be useful when it comes to comparing future pandemics and disease outbreaks, too.

\section{REFERENCES}

[1] Roy M. Anderson, T. Déirdre Hollingsworth, Rebecca F. Baggaley, Rosie Maddren, and Carolin Vegvari. 2020. COVID-19 spread in the UK: The end of the beginning? The Lancet 396, 10251 (2020), 587-590.

[2] Martin J. Conyon, Lerong He, and Steen Thomsen. 2020. Lockdowns and COVID-19 deaths in Scandinavia. Retrieved from SSRN 3616969 (2020).

[3] Matz Dahlberg, Per-Anders Edin, Erik Grönqvist, Johan Lyhagen, John Östh, Alexey Siretskiy, and Marina Toger. 2020. Effects of the COVID-19 pandemic on population mobility under mild policies: Causal evidence from Sweden. arXiv preprint arXiv:2004.09087 (2020).

[4] Ensheng Dong, Hongru Du, and Lauren Gardner. 2020. An interactive web-based dashboard to track COVID-19 in real time. Lancet. Infect. Dis. 20, 5 (May 2020), 533-534.

[5] Samuel Engle, John Stromme, and Anson Zhou. 2020. Staying at home: Mobility effects of COVID-19. DOI : https://doi.org/10.2139/ssrn. 3565703

[6] Charlie Giattino, Hannah Ritchie, Max Roser, Esteban Ortiz-Ospina, and Joe Hasell. 2020. Excess mortality during the Coronavirus pandemic (COVID-19). Retrieved from https://ourworldindata.org/excess-mortality-covid. 
[7] Rajan Gupta, Gaurav Pandey, Poonam Chaudhary, and Saibal K. Pal. 2020. Machine learning models for government to predict COVID-19 outbreak. Dig. Gov.: Res. Pract. 1, 4 (2020), 1-6.

[8] Thomas Hale, Anna Petherick, Toby Phillips, and Samuel Webster. 2020. Variation in government responses to COVID-19. Blavatnik School Gov. Work. Pap. 31 (2020).

[9] Andreas Kalk and Andreas Schultz. 2020. SARS-CoV-2 epidemic in African countries-Are we losing perspective? Lancet. Infect. Dis. (2020). DOI : https://doi.org/10.1016/S1473-3099(20)30563-6

[10] Munish Kumar, Surbhi Gupta, Krishan Kumar, and Monika Sachdeva. 2020. Spreading of COVID-19 in India, Italy, Japan, Spain, UK, US: A prediction using ARIMA and LSTM model. Dig. Gov.: Res. Pract. 1, 4 (2020), 1-9.

[11] Kai Kupferschmidt. 2020. The lockdowns worked-But what comes next? Science 6488 (2020), 218-219.

[12] Moustapha Mbow, Bertrand Lell, Simon P. Jochems, Badara Cisse, Souleymane Mboup, Benjamin G. Dewals, Assan Jaye, Alioune Dieye, and Maria Yazdanbakhsh. 2020. COVID-19 in Africa: Dampening the storm? Science 369, 6504 (2020), 624-626.

[13] Hannah Ritchie, Esteban Ortiz-Ospina, Diana Beltekian, Edouard Mathieu, Joe Hasell, Bobbie Macdonald, Charlie Giattino, and Max Roser. 2020. Mortality Risk of COVID-19. Retrieved from https://ourworldindata.org/mortality-risk-covid.

[14] Max Roser, Hannah Ritchie, Esteban Ortiz-Ospina, and Joe Hasell. 2020. Coronavirus pandemic (COVID-19). Retrieved from https://ourworldindata.org/excess-mortality-covid.

[15] Barry Smyth. 2020. Lockdowns \& rebounds: A data analysis of what happens next. Dig. Gov: Res. Pract. 1, 4 (Aug. 2020). DOI : https:// doi.org/10.1145/3411762

[16] Robert Verity, Lucy C. Okell, Ilaria Dorigatti, Peter Winskill, Charles Whittaker, Natsuko Imai, Gina Cuomo-Dannenburg, Hayley Thompson, Patrick G. T. Walker, Han Fu, Amy Dighe, Jamie T. Griffin, Marc Baguelin, Sangeeta Bhatia, Adhiratha Boonyasiri, Anne Cori, Zulma Cucunubá, Rich FitzJohn, Katy Gaythorpe, Will Green, Arran Hamlet, Wes Hinsley, Daniel Laydon, Gemma NedjatiGilani, Steven Riley, Sabine van Elsland, Erik Volz, Haowei Wang, Yuanrong Wang, binfopersonXiaoyue Xi, Christl A. Donnelly, Azra C. Ghani, and Neil M. Ferguson. 2020. Estimates of the severity of coronavirus disease 2019: A model-based analysis. Lancet. Infect. Dis. 20, 6 (June 2020), 669-677.

[17] Russell M. Viner, Simon J. Russell, Helen Croker, Jessica Packer, Joseph Ward, Claire Stansfield, Oliver Mytton, Chris Bonell, and Robert Booy. 2020. School closure and management practices during coronavirus outbreaks including COVID-19: A rapid systematic review. Lancet Child Adoles. Health (2020). DOI : https://doi.org/10.1016/S2352-4642(20)30095-X

[18] Jing Yuan, Minghui Li, Gang Lv, and Z. Kevin Lu. 2020. Monitoring transmissibility and mortality of COVID-19 in Europe. Int. 7. Infect. Dis. 20 (2020), 1878:3511.

[19] Juanjuan Zhang, Maria Litvinova, Yuxia Liang, Yan Wang, Wei Wang, Shanlu Zhao, Qianhui Wu, Stefano Merler, Cécile Viboud, Alessandro Vespignani, Marco Ajelli, and Hongjie Yu. 2020. Changes in contact patterns shape the dynamics of the COVID-19 outbreak in China. Science 368, 6498 (2020), 1481-1486.

[20] Sheng Zhang, Meng Yuan Diao, Liwei Duan, Zhaofen Lin, and Dechang Chen. 2020. The novel coronavirus (SARS-CoV-2) infections in China: Prevention, control and challenges. Intens. Care Med. 46, 4 (Apr. 2020), 591-593.

Received September 2020; revised November 2020; accepted November 2020

Digital Government: Research and Practice, Vol. 2, No. 2, Article 21. Publication date: December 2020. 